\title{
Real-time Strehl and image quality performance estimator at Paranal observatory
}

\author{
Dimitri Mawet ${ }^{a}$, Alain Smette ${ }^{a}$, Marc S. Sarazin ${ }^{b}$, Harald Kuntschner ${ }^{b}$, and Julien H. Girard ${ }^{a}$ \\ ${ }^{a}$ European Southern Observatory, Alonso de Córdova 3107, Vitacura, Casilla 19001, Chile; \\ ${ }^{b}$ European Southern Observatory, Karl-Schwarzschild-Str., Garching bei München, Germany;
}

\begin{abstract}
Here we describe a prototype Strehl and image quality performance estimator and its integration into Paranal operations, starting with UT4 and its suite of three infrared instruments: adaptive optics-fed imager/spectrograph NACO (temporarily out of operations) and integral field unit SINFONI, as well as wide-field imager HAWKI. The real-time estimator processes the ambient conditions (seeing, coherence time, airmass, etc.) from the DIMM, and telescope Shack-Hartmann image analyzer to produce estimates of image quality and Strehl ratio every $\sim 30$ seconds. The estimate is using ad-hoc instrumental models, based in part on the PAOLA adaptive optics simulator. We discuss the current performance of the estimator vs real IQ and Strehl measurements, its impact on service mode efficiency, prospects for full deployment at other UTs, its use for the adaptive optics facility (AOF), and inclusion of the SLODAR-measured fine turbulence characteristics.
\end{abstract}

Keywords: Adaptive Optics, Very Large Telescope, Strehl ratio, Observation scheduling, Quality Control, Science operations

\section{INTRODUCTION}

The advent of widespread adaptive optics (AO) at major observatories is ineluctable. The Very Large Telescope (VLT) in Chile will soon operate a park of 6 instruments equipped with adaptive optics modules, without even counting the current and next-generation adaptive optics systems feeding the Very Large Telescope Interferometer (VLTI) instruments. The complexity of AO instruments and their sensitivity to atmospheric conditions (e.g. turbulence strength and speed) calls for an overhaul of operational practices relying on the sole seeing and transparency conditions for observing blocks scheduling and, to a lesser extent classification.

ESO's service mode operations scheme and the availability of 3 instruments at all times on each one of the four 8.2-meter unit telescopes (UT) is one of the strengths of the VLT, and likely one of the reasons of its scientific success. Scheduling of service mode observing blocks (OBs) is ultimately the responsibility of the astronomer and telescope instrument operator, which have to judge the matching of current conditions to the set of constraints set by the end users. For spectroscopy and seeing limited operations, this can be done on the fly thanks to powerful ambient site monitoring tools (ESO's ASM keeps track of temperature at various heights, humidity, atmospheric pressure, dew point, seeing, coherence time, water vapor, sky infrared luminosity, transparency, wind speed at various heights, etc.).

For adaptive optics system, since the user constraint is Strehl ratio rather than image quality, the conversion from classical monitoring tools to this metric is far from obvious, and requires a deep knowledge of the instrument and the response of the requested mode to specific ambient conditions such as seeing $\theta_{0}$, coherence time $\tau_{0}$, turbulence profile, etc. These relationship are non-linear, and hard to guess, even for veteran astronomers and operators.

Here we present the Paranal Strehl ratio Monitor (PSM), which predicts in real-time the potential Strehl ratio under current conditions as monitored by Paranal ambient site monitoring tools, and the image quality measured by the VLT Unit Telescope Shack-Hartmann image analyzer (used for cophasing the primary mirror).

Further author information: send correspondence to dmawet@eso.org 


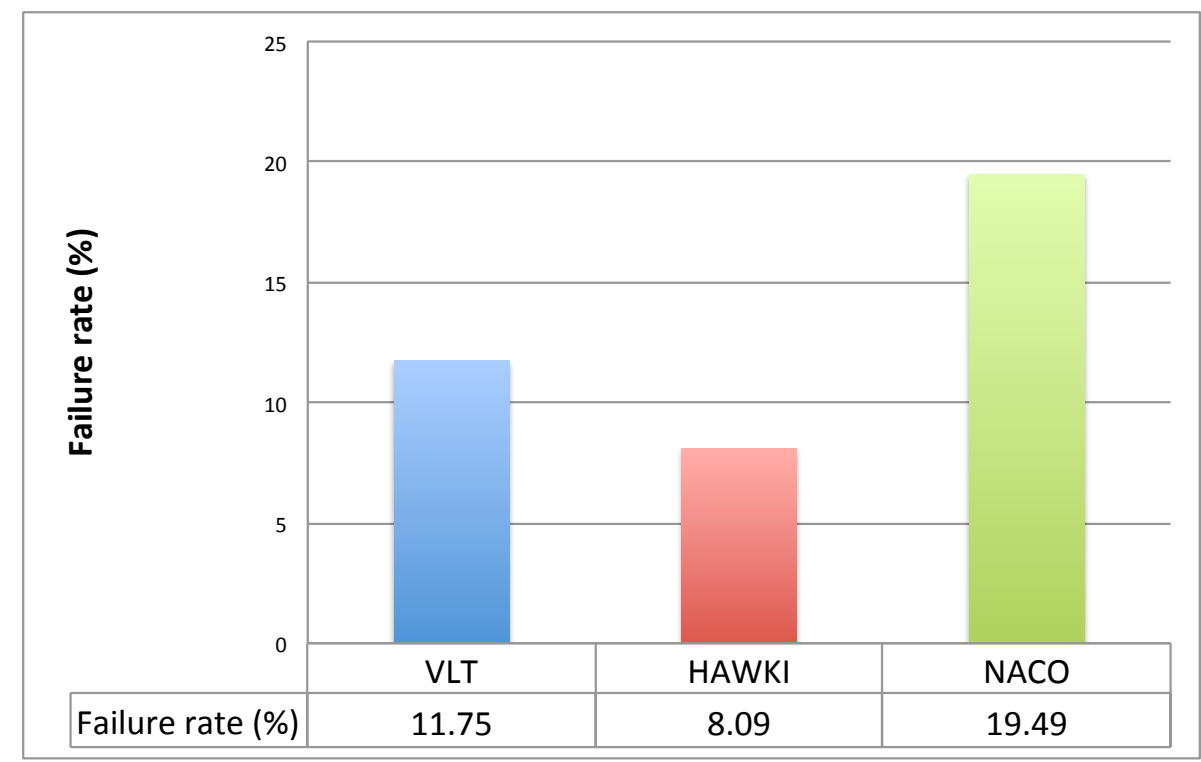

Figure 1. Observing block failure rate for a representative adaptive optics instrument, here NACO, and a near-infrared seeing-limited imager, here HAWK-I, and the average for the VLT instruments. These statistics are based on 2005-2010 VLT night logging data (source: M. F. Sterzik).

\section{GOALS OF THE PSM: OPTIMIZE SCHEDULING AND CLASSIFICATION}

The goal of the monitor/predictor is twofold. First, it allows the user to start AO observing blocks with a much better confidence over their final quality. Second, for difficult cases where direct measurements are precluded (e.g. extended objects), it helps the quality control and subsequent classification.

\subsection{Failure rate of $\mathrm{AO}$ observations in service mode}

Service mode operations at the VLT represent $\simeq 60 \%$ of the total telescope time. Observing block classification is performed according to a 4-step scale: A - fully within specifications, B - mostly within specifications, C - out of specs, to be repeated, D - out of specs, do not repeat. The pool of constraints used to perform classification include: Seeing, Sky Transparency, Airmass, FLI, Moon Distance, Twilight, Water Vapour, and finally Strehl ratio for AO instruments. Note that each VLT instrument uses a subset of these constraints, following the relevance with their respective science.

The failure rate of $\mathrm{OB}$ is hereby defined as the number $\mathrm{C}$ and $\mathrm{D}$ classified OBs over all service mode OBs (A, B, C, D), i.e. $F R=(C+D) /(A+B+C+D)$. It is primarily related to the scheduling efficiency, itself a function of the capability of matching best the requested observing constraints with the current conditions. This matching is straightforward for classical constraints, even though some of them require dedicated measurements (involving special apparatus such as radiometers, DIMM/MASS/SLODAR stations, etc.).

Strehl ratio, currently used by NACO and SINFONI as a constraint and quality control parameter, is a particular metric that requires its own treatment. First of all, its measurement is not straightforward. Second, predicting Strehl based on atmospheric conditions require complicated models involving an accurate representation of the adaptive optics system and the instrument attached to it.

\subsection{Quality control}

Quality control of AO observations is a non-trivial task (see Girard et al. 2014, these proceedings). Accurately measuring Strehl ratio on real-life data is difficult. ${ }^{1}$ Typical error bars on Strehl ratio measurement are of the order of $5-10 \%$. In some cases (e.g. extended objects, very faint objects, coronagraphy, etc.), any direct measurement is precluded. Objective assessment of image quality through robust indirect methods is therefore needed. 


\section{Paranal Strehl Monitor Architecture}

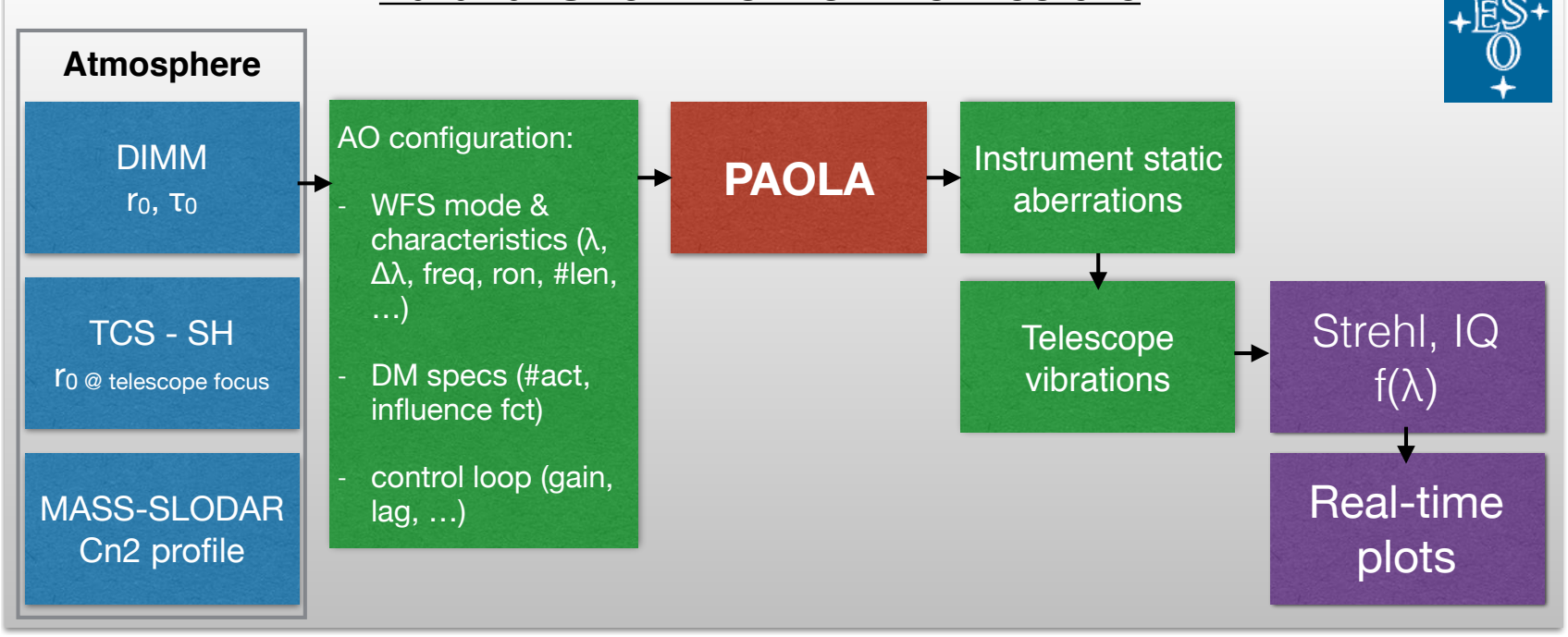

Figure 2. Architecture of the Paranal Strehl predictor. Note that the DIMM does not measure $\tau_{0}$ directly but extrapolates it from the $r_{0}$ and wind speed measured at ground level or predicted at $200 \mathrm{mb}$ (jet stream altitude) by a meteorological model.

\section{ARCHITECTURE OF THE PREDICTOR}

One of the requirements for the PSM it to use currently available Paranal site monitoring infrastructure, and be flexible enough to accommodate future ones, such as SLODAR and telemetric data from various wavefront sensors coming to Paranal in the future. The architecture, presented in the block diagram of Fig. 2, is currently based on inputs from the DIMM, Telescope Control System active optics Shack-Hartmann wavefront sensor, a model of the AO system of NACO and SINFONI, along with empirical error terms for internal static aberrations and telescope vibrations.

The core of the predictor is PAOLA, which stands for Performance of Adaptive Optics for Large (or Little) Apertures. ${ }^{2}$ PAOLA is a set of functions and procedures written in IDL for modeling the performances of an astronomical adaptive optics (AO) system. Unlike most AO simulation packages, PAOLA uses a synthetic approach, where the general behavior of the whole system is modeled. The core of the method is based on an analytic expression for the residual phase spatial power spectrum (PSD, which is a statistical quantity), and its relationship with the optical transfer function (OTF), from which the AO system PSF can be simulated. The main advantage over the Monte-Carlo approach is the enormous gain in computation time which allows deriving critical AO parameters in a couple of seconds, instead of hours, or days, which is well-suite to the needs of a real-time monitor.

\subsection{Modeling NACO}

$\mathrm{NACO}^{3}$ has been ESO's workhorse adaptive optics infrared camera and spectrograph since its installation and commissioning in 2002 (see Girard et al. 2014, these proceedings). This highly successful instrument has been temporarily decommissioned last year to make space for MUSE on UT4. NACO will be back on sky in late 2014, and refurbished in 2015, to prolong its life for hopefully many years. NAOS, the Nasmyth Adaptive Optics System, ${ }^{3}$ the AO module of the coronagraphic infrared camera CONICA, consists of a 185-actuator deformable mirror manufactured by CILAS, and a tip-tilt mirror manufactured by Observatoire de Paris. To command these two corrective elements, NAOS is equipped with 2 wavefront sensor arms, functioning the visible and infrared wavelength, respectively.

The simulation of NACO in PSM includes detailed characteristics of the DM, and wavefront sensors, such as number of actuators, influence function, wavelength range, and sensor frequency and readout noise. Closed loop behavior (including loop lag) and realistic gains are also sent to PAOLA's engine. The model and its 


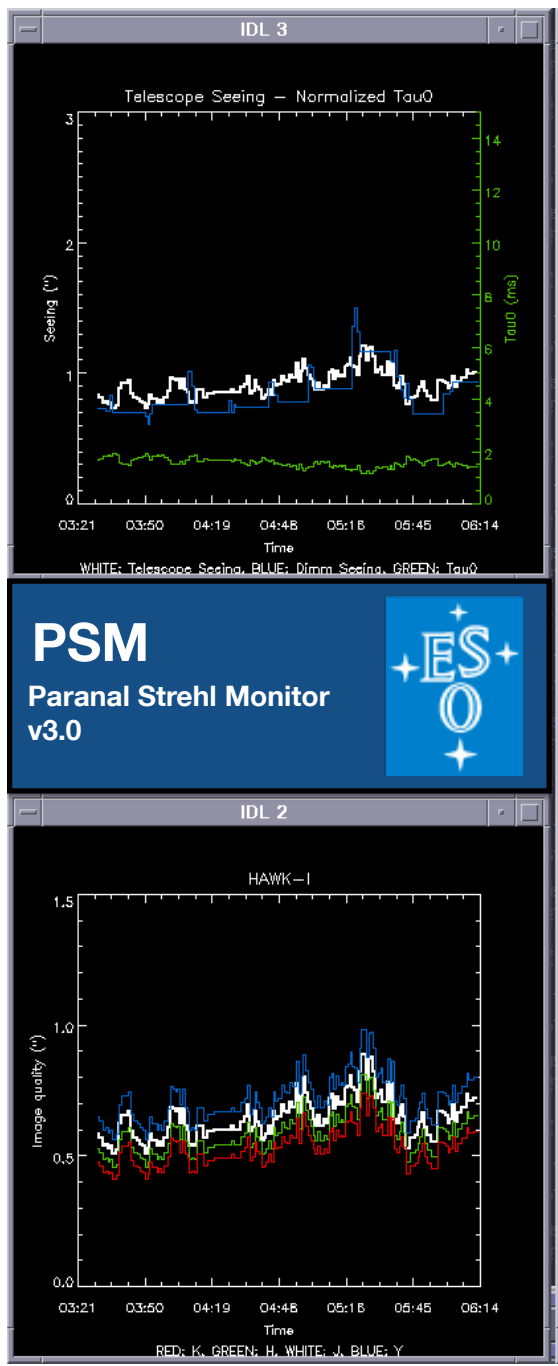

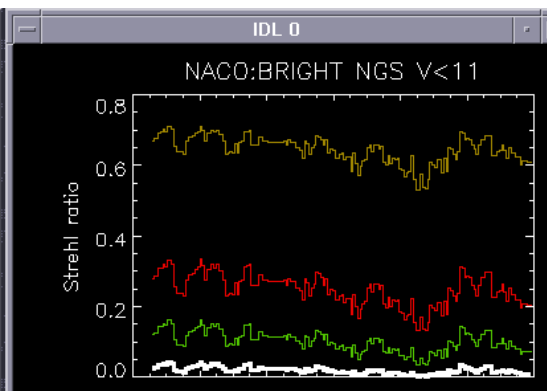

$\begin{array}{llllllll}03: 21 & 03: 50 & 04: 19 & 04: 48 & 05: 16 & 05: 45 & 06: 14\end{array}$ Time

ORA: L, RED: K, GREEN: H, WHITE: J NACO:AVERAGE NGS $11<V<13$

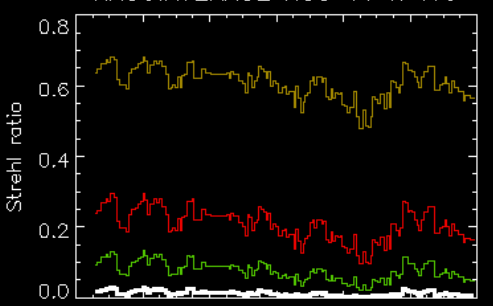

$\begin{array}{lllllll}03: 21 & 03: 50 & 04: 19 & 04: 48 & 05: 16 & 05: 45 & 06: 14\end{array}$ Time ORA: L, RED: K, GREEN: H, WHITE: ل NACO:FAINT NGS $13<\mathrm{V}$

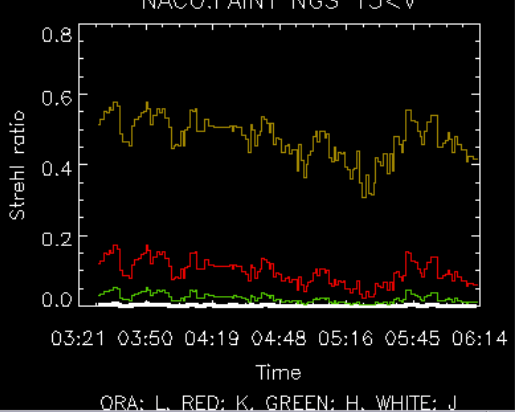

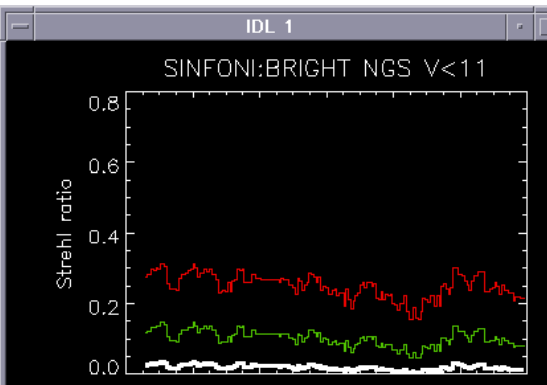

$\begin{array}{lllllll}03: 21 & 03: 50 & 04: 19 & 04: 48 & 05: 16 & 05: 45 & 06: 14\end{array}$ Time

RED: K, GREEN: H, WHITE: J SINFONI:AVERAGE NGS $11<V<13$

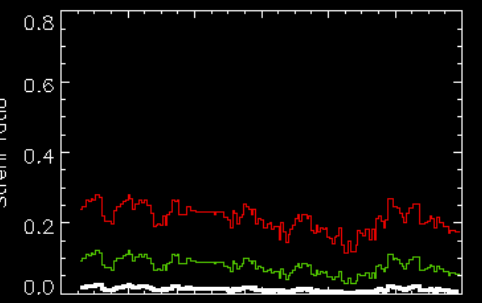

$\begin{array}{lllllll}03: 21 & 03: 50 & 04: 19 & 04: 48 & 05: 16 & 05: 45 & 06: 14\end{array}$ Time RED: K, GREEN: H, WHITE: J SINFONI:FAINT NGS $13<\mathrm{V}$

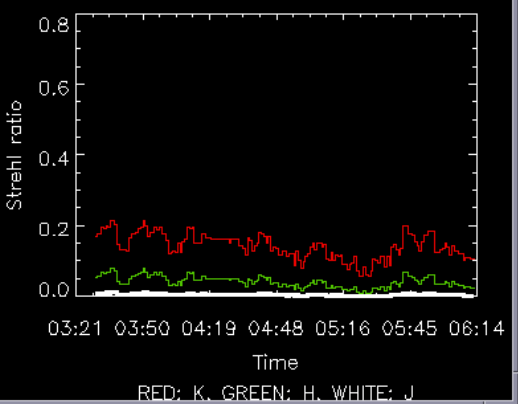

Figure 3. PSM dashboard. The upper left window shows the seeing as measured by DIMM and TCS-SH, as well as the coherence time at the telescope level. The lower left window shows the image quality for HAWK-I in Y, J, H, and K bands. The central column shows the Strehl ratio estimate for NACO, in the faint, median, and bright natural guide star regimes, for J, H, K, and L bands. The right column shows the Strehl ratio estimates for SINFONI, in the faint, median, and bright natural guide star regimes, for $\mathrm{J}, \mathrm{H}$ and $\mathrm{K}$ bands.

detailed parameters have been fine tuned against real data in various conditions, using RTC estimates and Strehl measurement tools such as the MAD Strehl Meter, ${ }^{4}$ and ABISM (Girard et al. 2014, these proceedings). The relative accuracy of the prediction has been measure to be of the order of $\pm 5 \%$ in various seeing conditions. This accuracy is more than sufficient for ESO's service mode classification guidelines at Paranal observatory.

\subsection{Modeling SINFONI}

SINFONI is a near-infrared integral field spectrograph fed by a curvature sensing adaptive optics module. PAOLA does not support this kind of wavefront sensing mode, so we instead used an ad-hoc representation to model the SINFONI MACAO module. The model parameters were also fine tuned and the output validated against real data. The predictor relative accuracy has been measured to be of the order of $\pm 10 \%$ in various seeing conditions.

\subsection{Modeling HAWK-I}

The modeling of wide-filed infrared imager HAWK-I is straightforward since it is seeing limited. A wavelength scaling is performed on the seeing as seen by the TCS Shack-Hartmann active optics wavefront sensor. 


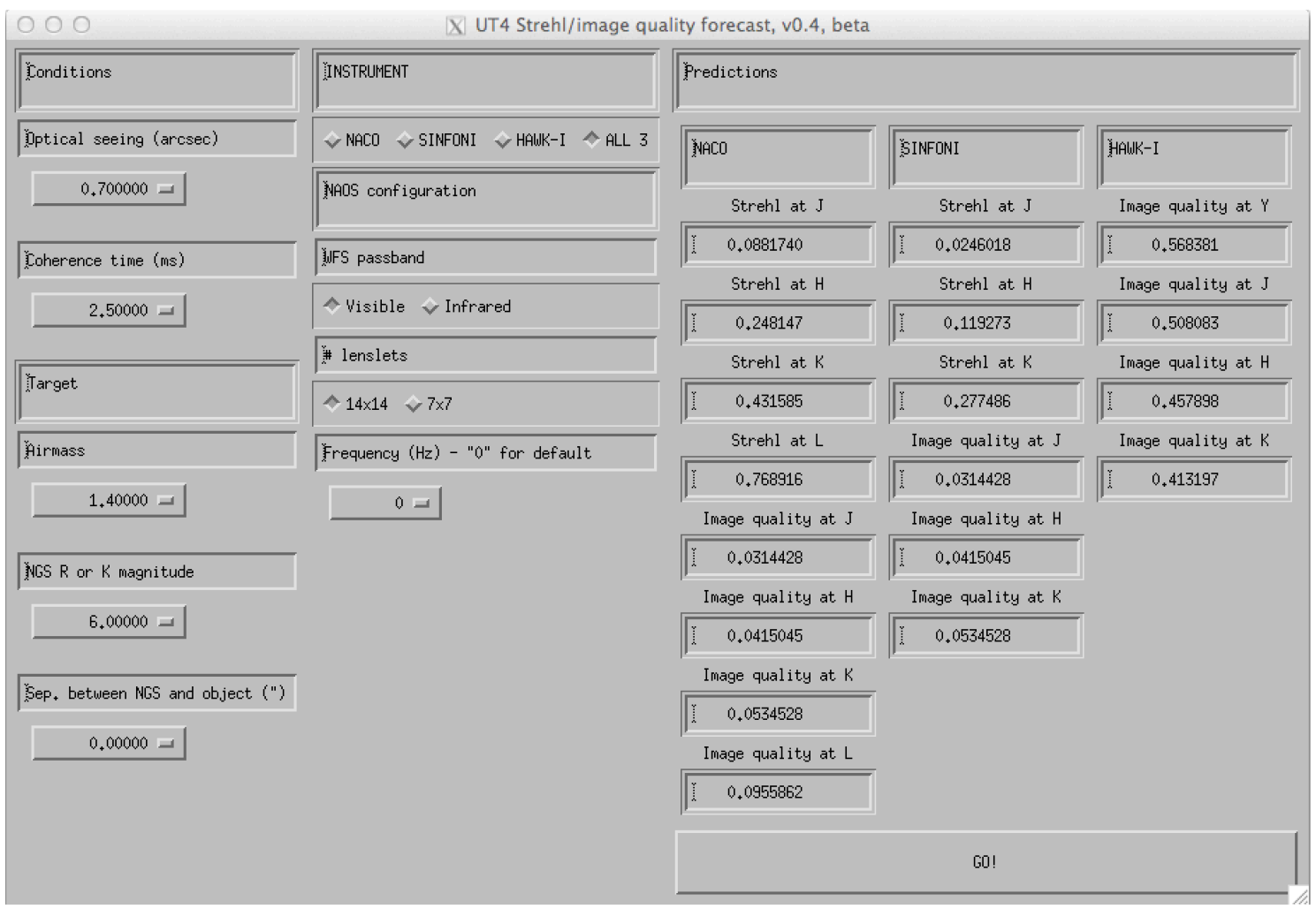

Figure 4. PSM dashboard in manual expert mode.

\section{PSM MODES}

The PSM has two modes. The main mode is a fully automatic, monitoring mode displaying quasi real-time plots. There is also manual expert mode to explore more parameters. It is also the only way to precisely evaluate the impact of airmass or off-axis NGS, and also roughly predict Strehl for Laser Guide Star (LGS) AO.

\subsection{Monitoring, automatic mode}

The monitoring mode aims at providing the user with a set of temporal series plots showing the evolution of a subset of well-chosen extrapolated Strehl ratios and image quality parameters of all relevant instruments at once (see Fig. 3). The instrument setups chosen for this real-time simulation are the most common ones (major broadband filters). The natural guide star brightness (NGS), a critical parameter for AO performance, is a pre-selection for three AO-correction regimes corresponding to faint, median, and bright NGS, so a total of 3 plots are generated for each $\mathrm{AO}$ instrument (see Fig. 3). The plots are updated with new values at the frequency of the VLT active optics refresh rate, i.e. every 30-60 seconds.

\subsection{Expert, manual mode}

The expert mode (see Fig. 4) allows manually entering the ambient conditions (seeing, coherence time), target characteristics (magnitude, airmass) and configuration of the instrument adaptive optics (separation between NGS, visible or IR wavefront sensor, number of lenslets, and frequency). It also enables selecting which instrument to consider (if one only needs to compute quality control quantities for a single instrument).

The expert mode GUI is currently implemented in IDL and calls PAOLA for immediate computation of relevant output parameters: Strehl ratio, and image quality in arcseconds. 


\section{CONCLUSIONS AND FUTURE WORK}

The tool, currently at version 3.0 is fully functional, called with a one-line command from the offline machine used by night-time astronomers and operators. Its integration in the operation scheme at Paranal is very straightforward. Because of the recent removal of NACO, the main adaptive optics instrument of UT4, not enough experience has been gained to measure its impact on observing efficiency yet. User feedback is encouraging though, both for the scheduling and classification aspects of Paranal operations.

Future developments include the following:

1. Migration to Python, and precomputation of Strehl ratio results on fine (seeing, coherence) grids with PAOLA (or any other simulation tool) once and for all, in order to increase efficiency and speed.

2. Time series forecasting, i.e. the use of a model to predict future values based on previously observed values, in an attempt to truly anticipate performance on the timescale of a few hours, and integration in the Paranal VLT observing scheduling tool (OT).

3. Integration at all Paranal UTs, starting with new AO modules such as SPHERE (under commissioning, a prototype of the predictor was tested during the first light of SPHERE, Beuzit et al. 2014, these proceedings)

4. Adaptation to the Adaptive Optics facility, implementing ground-layer adaptive optics mode (GLAO).

The Adaptive Optics Facility (AOF) aim is to upgrade UT4 with an adaptive secondary mirror. The goal is to make this telescope an Adaptive Telescope providing turbulence corrected images at all focii, without the addition of adaptive modules and supplementary optics in front of the instruments (each instrument will have its own AO module: GRAAL for HAWK-I, GALACSI for MUSE, ERIS will have its own pyramid wavefront sensor). The concept is far more reaching than only a deformable secondary mirror (DSM) since the instrument park (HAWK-I, MUSE, and later ERIS) is optimized to benefit from this upgrade. Several novel adaptive correction modes are also envisioned making use of a sophisticated Laser launch facility that will provide 4 LGS for wavefront sensing (for more details, see Arsenault et al. 2014, these proceedings).

\section{ACKNOWLEDGMENTS}

This work was carried out at the European Southern Observatory (ESO) site of Paranal, Vitacura (Santiago, Chile), and Garching (Germany).

\section{REFERENCES}

[1] Roberts, Jr., L. C., Perrin, M. D., Marchis, F., Sivaramakrishnan, A., Makidon, R. B., Christou, J. C., Macintosh, B. A., Poyneer, L. A., van Dam, M. A., and Troy, M., "Is that really your Strehl ratio?," in [Advancements in Adaptive Optics], Bonaccini Calia, D., Ellerbroek, B. L., and Ragazzoni, R., eds., Society of Photo-Optical Instrumentation Engineers (SPIE) Conference Series 5490, 504-515 (Oct. 2004).

[2] Jolissaint, L., Véran, J.-P., and Conan, R., "Analytical modeling of adaptive optics: foundations of the phase spatial power spectrum approach," Journal of the Optical Society of America A 23, 382-394 (Feb. 2006).

[3] Rousset, G., Lacombe, F., Puget, P., Hubin, N. N., Gendron, E., Fusco, T., Arsenault, R., Charton, J., Feautrier, P., Gigan, P., Kern, P. Y., Lagrange, A.-M., Madec, P.-Y., Mouillet, D., Rabaud, D., Rabou, P., Stadler, E., and Zins, G., "NAOS, the first AO system of the VLT: on-sky performance," in [Adaptive Optical System Technologies II], Wizinowich, P. L. and Bonaccini, D., eds., Society of Photo-Optical Instrumentation Engineers (SPIE) Conference Series 4839, 140-149 (Feb. 2003).

[4] Marchetti, E., Brast, R., Delabre, B., Donaldson, R., Fedrigo, E., Frank, C., Hubin, N., Kolb, J., Lizon, J.-L., Marchesi, M., Oberti, S., Reiss, R., Soenke, C., Tordo, S., Baruffolo, A., Bagnara, P., Amorim, A., and Lima, J., "MAD on sky results in star oriented mode," in [Society of Photo-Optical Instrumentation Engineers (SPIE) Conference Series], Society of Photo-Optical Instrumentation Engineers (SPIE) Conference Series 7015 (July 2008). 